\title{
Graphene Logo Patch Antenna
}

\author{
Varindra Kumar $^{1}$
}

\begin{abstract}
Graphene with its unique electrical, mechanical and physical properties provides a platform for novel electronic systems design. A compact patch Logo antenna based on doped Graphene has been proposed for its operation within GHz range. The antenna parameters such as S11 parameter, Bandwidth, Gain, Directivity and Efficiency has been obtained and compared with results obtained from a copper conductive patch antenna. The designed antenna has an elliptical shape with a dimension of $25 \times 16 \mathrm{~mm}$. It has been designed over $1.6 \mathrm{~mm}$ thick polyimide substrate to provide the flexibility required for applications ranging from wearable to medical electronics. Various parametric plots for Graphene such as dispersion diagram, conductivity as a function of its DC bias, surface impedance and comparison of skin depth with copper conductive media have been obtained for its inclusion in $3 D$ field solver tool. Finally a spice circuit has been obtained for the S11 parameter of the graphene and copper conductive patch antenna for its $3 D$ field solver equivalence.
\end{abstract}

Keywords: Antenna, Copper, GHz, Graphene, Logo, Patch, Spice

\section{Nomenclature}

e Electron's constant

$\mathrm{f}_{\mathrm{d}}(\epsilon) \quad$ Fermi Dirac distribution function

$\mathrm{FeCl}_{3} \quad$ Ferric Chloride

h Planck's constant

h Reduced Planck's constant

IPC Institute for Printed Circuits

$\mu_{\mathrm{C}} \quad$ Chemical potential

$\mathrm{k}_{\mathrm{B}} \quad$ Boltzmann constant

$\mathrm{n}_{\mathrm{s}} \quad$ electron's mobility

$\Omega \quad$ Ohm

PEC Perfect Electric Conductor

RLC Resistor, Inductor and Capacitor

$\sigma \quad$ Conductivity

$\sigma_{0} \quad$ Fixed Conductivity

$\sigma_{\mathrm{s}} \quad$ Surface Conductivity

$\mathrm{T} \quad$ Room temperature

$\tau \quad$ Relaxation Time constant

$\mathrm{V}_{\mathrm{f}} \quad$ Fermi velocity

VSWR Voltage Standing Wave Ratio

$\omega$

Operating Frequency

\section{Introduction}

With the arrival of new generation electronic devices and its miniaturization there is a continuous drive for various components within the electronic system to be small, compact, flexible and still it has to provide sturdiness. The antenna is one element of these modern electronic systems and provides communication link within and across components. Due to limited bandwidth availability in some selective $\mathrm{GHz}$ bands such as $2.4 \mathrm{GHz}$ and $5 \mathrm{GHz}$ and with an increase of new generation electronic devices, communication systems are moving towards still untapped higher $\mathrm{GHz}$ frequency range. With an increase in the operating frequency, the frequency band can provide a wider bandwidth and a higher data rate transfer mechanism over a secure channel. At the same time, an increase in operating frequency range itself provides an opportunity to design small and miniaturised systems however the resonant structures at higher frequency range cause severe attenuation with reduced electron mobility. Graphene with its high mobility and somewhat comparable conductivity within its upper $\mathrm{GHz}$ and $\mathrm{THz}$ range provides an alternate mechanism to improve the drawback faced by traditional metallic structures for antenna applications. The frequency dependent conductivity of the single layer graphene material although is comparable to copper in its $\mathrm{THz}$ range, it is not very good in $\mathrm{GHz}$ range [1 ].

A multi-layer $\mathrm{FeCl}_{3}$ doped Graphene conductive material [2], [3] based $\mathrm{GHz}$ antenna over polyimide substrate has been designed in this paper and various antenna parameters such as S11 parameter, VSWR, Bandwidth, Gain and efficiency has been plotted for its comparison to copper conductive patch. The ground layer is designed using PEC (Perfect Electric Conductor). As the graphene flake can be biased with a DC voltage and this function can provide a switching resonance at the desire, the conductivity and surface impedance plot for various DC voltage bias have been obtained here. The DC voltage bias may provide a technique for manual or automatic configuration of the patch resonance. On the other hand, a polyimide substrate can provide flexibility in the shape with its application in healthcare, military and wearable domain. The paper has been divided into the following four sections with Introduction, Design methodology, results and discussion, and finally conclusion. Design methodology describes the Graphene conductivity and surface impedance behavior within $\mathrm{THz}$ and $\mathrm{GHz}$ range, patch antenna design, the 
method to obtain spice circuit equivalence and its comparison to full field solver tool while the results and discussion section talks about the comparison of various antenna parameters for graphene and copper patch.

\section{Design Methodology}

As this paper describes about the graphene and copper conductive media for the Logo antenna design, the graphene properties such as dispersion diagram, conductivity, its surface impedance and skin depth is obtained and plotted using Matlab. The antenna has been designed using CST (a 3D field solver tool). The spice circuit, which can provide an equivalent electrical circuit, has been derived using ADS tool with importing the S11 parameter from CST.

\section{II.1. Graphene}

The discovery of graphene and its subsequent development for various electronic applications [4] - [6] has made it quite attractive element in the area of wide ranging applications. The $\mathrm{Sp}^{2}$ bond of the Graphene even after being one atom thick contains unique thermal, mechanical, electrical and chemical behavior and even after forming a wide surface area it maintains consistent and reliable properties within its operating region. With its 2D nanostructure properties, it can be stacked and combined to make 3D layered structure for various applications ranging from novel sensor design to RF or optical material design. Recently various types of graphene and graphene based hybrid (alloy with other elements such as polymer, oxide, metallic mixtures) have been designed and developed to enhance these physical and electrical properties further [7]. This paper talks about the design of Graphene based patch antenna for its operation within $\mathrm{GHz}$ range and its comparison with copper conductive media. The graphene based 2D layered structure shows a similar electrical conductivity to other conductive materials or metals within $\mathrm{THz}$ range and there have been consistent efforts to increase the conductivity of its structure using metallic or polymerized mixture in $\mathrm{GHz}$ range. A high electron mobility in the range of $200,000 \mathrm{~cm}^{2} / \mathrm{V}$-s, thermal conductivity of $5000 \mathrm{~W} / \mathrm{m} / \mathrm{K}$, surface area of $2630 \mathrm{~m}^{2} / \mathrm{g}$ and Young's modulus of $1100 \mathrm{GPa}$ also makes graphene an ideal choice for various applications [8].

Figs. 1 and 2 show the graphene honeycomb lattice structure in $2 \mathrm{D}$ and $3 \mathrm{D}$ form. The electrical properties of the structure can be derived after understanding the dispersion relationship of its structure. The dispersion relation of the graphene flake with $\mathrm{t}=2.7 \mathrm{~V}$ and $\mathrm{t}^{\prime}=0 \mathrm{~V}$ can be defined using (1) and (2) and a Matlab plot has been obtained and shown in Fig. 3 [9] - [14]. Here six convergence points representing the graphene bond show the fermi level with low conductivity at its Fermi Dirac points (also known as charge neutrality points). The dispersion diagram is used to show the performance of the conductivity and sensitivity for a Graphene flake.

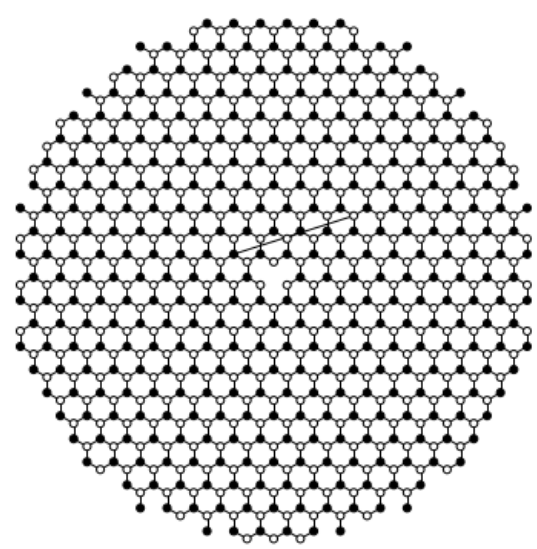

Fig. 1 2D Graphene structure

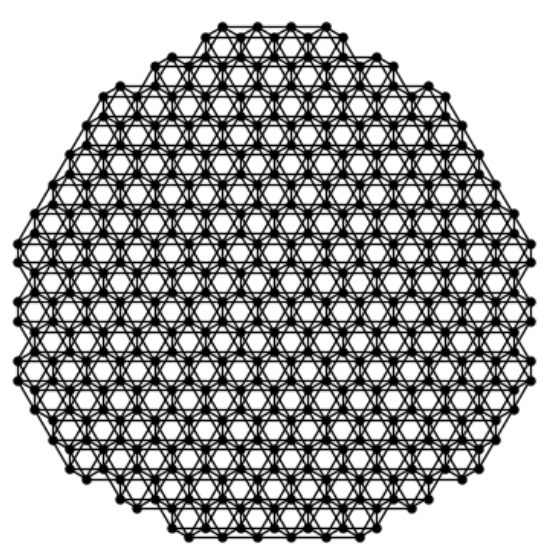

Fig. 2 3D Graphene structure

$$
E(k)= \pm t \sqrt{3+f(k)}-t^{\prime} f(k)
$$

$f(k)=2 \cos \left(\sqrt{3} a k_{y}\right)+4 \cos \left(\frac{\sqrt{3}}{2} a k_{y}\right) \cos \left(\frac{3}{2} a k_{x}\right)(2)$

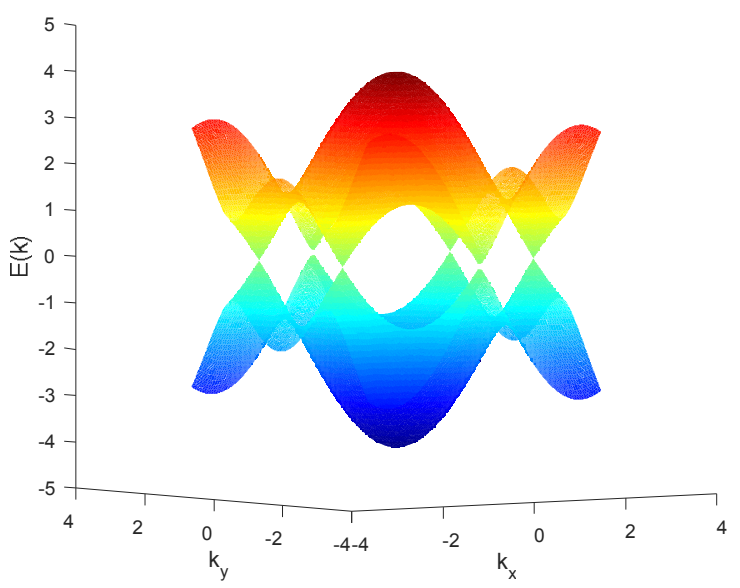

Fig. 3 Fermi energy Dispersion diagram 
In the absence of any externally applied electric and magnetic field, the graphene conductivity can be defined using Kubo's equation [15] - [17] and is represented as (3). Here $\omega$ is the operating frequency, $\mu_{C}$ is the applied chemical potential to bias the graphene structure, $\tau$ is the relaxation time constant (with $1 / \tau$ defining the carrier scattering rate), T being the room temperature and $f_{d}(\epsilon)$ is the Fermi Dirac distribution function while $k_{B}$ is the Boltzmann constant, $\hbar$ is the reduced Planck's constant and $\mathrm{V}_{\mathrm{f}}$ is the Fermi velocity of the photon. The Fermi Dirac function $f_{d}(\epsilon)$, electron mobility $n_{s}$, relaxation time $\tau$ and the chemical potential $\mu_{\mathrm{C}}$ can be represented using (4), (5), (6) and (7). For a pure Graphene flake where $\mu_{\mathrm{C}}>\mathrm{k}_{\mathrm{B}} \mathrm{T}$, the carrier mobility can be defined using (5) and with an approximation in the Fermi Dirac distribution function it can be solved as (8) however with the graphene being highly doped with other materials, the relationship $\mu_{\mathrm{C}}<\mathrm{k}_{\mathrm{B}} \mathrm{T}$ gets satisfied and the carrier mobility is defined using (9).

$$
\begin{aligned}
& \sigma\left(\omega, \mu_{C}, \tau, T\right)=\frac{j e^{2} / \pi \hbar^{2}}{\omega-j(2 / \tau)} \\
& \times \int_{0}^{\infty} \varepsilon\left[\frac{\partial f_{d}(\varepsilon)}{\partial \varepsilon}-\frac{\partial f_{d}(-\varepsilon)}{\partial \varepsilon}\right] d \varepsilon \\
& +j e^{2}\left(\omega-j(2 / \tau) / \pi \hbar^{2}\right. \\
& \times \int_{0}^{\infty} \frac{f_{d}(\varepsilon)-f_{d}(-\varepsilon)}{(\omega-j(2 / \tau))^{2}-4(\varepsilon / \hbar)^{2}} d \varepsilon \\
& f_{d}(\varepsilon)=\left[1+e^{\frac{\varepsilon+\left|\mu_{C}\right|}{k_{B} T}}\right]^{-1} \\
& n_{s}=\frac{2}{\pi \hbar^{2} V_{f}^{2}} \int_{0}^{\infty} \varepsilon\left[\begin{array}{l}
f_{d}\left(\varepsilon-\mu_{C}\right)- \\
f_{d}\left(\varepsilon+\mu_{C}\right)
\end{array}\right] d \varepsilon \\
& \tau=\frac{\mu \mu_{C}}{e V_{f}^{2}} \\
& \mu_{C}=\sqrt{\pi \hbar^{2} V_{f}^{2} n_{s}} \\
& n_{s}=\frac{2}{\pi \hbar^{2} V_{f}^{2}}\left(k_{B} T\right)^{2}\left(e^{\frac{2 \mu_{C}}{k_{B} T}-1}\right) \\
& n_{s}=\frac{\mu_{C}^{2}}{\pi \hbar^{2} V_{f}^{2}}
\end{aligned}
$$

The solution of an integral for (3) provides a combined conductivity with the effect of carrier contribution. Here the first part of (3) provides the intraband conductivity and can be represented as (10) while the later part defines the interband conductivity and can be represented as (11). $\sigma_{0}$ can be presented as $\pi \mathrm{e}^{2} /(2 \mathrm{~h})$ and is used to normalize the conductivity [18]. These relations indicate that the graphene mobility or conductivity can be controlled using an external bias voltage $V_{D C}$ which can change the conductivity of the graphene flake. Many research works have been published in the past for Graphene applications within the THz frequency region [19] - [21] and few works have been presented in GHz region [22] - [24]. The work in this paper presents an application of graphene patch within $\mathrm{GHz}$ range and its comparison to the copper conductor for an antenna patch behavior.

$$
\begin{aligned}
& \sigma_{\text {int } r a}\left(\omega, \mu_{C}, \tau, T\right)=j \frac{8 \sigma_{0} k_{B} T / h}{\omega-j 2 / \tau} \\
& \times\left[\frac{\mu_{C}}{k_{B} T}+2 \ln \left(e^{-\frac{\mu_{C}}{k_{B} T}}+1\right)\right] \\
& \sigma_{\text {int } e r}\left(\omega, \mu_{C}, \tau, T\right)=-j \frac{\sigma_{0}}{\pi} \\
& \times\left[\frac{2\left|\mu_{C}\right|-(\omega-j 2 / \tau) \hbar}{2\left|\mu_{C}\right|+(\omega-j 2 / \tau) \hbar}\right] \\
& \delta=\sqrt{\frac{2}{\omega \mu \sigma}}
\end{aligned}
$$

The normalized conductivity plot for its operating frequency range from radio wave $(10 \mathrm{MHz})$ to visible light wave $(500 \mathrm{THz})$ has been obtained using Matlab and is shown in Fig. 4. Here the conductivity is normalized with its constant conductivity term $\sigma_{0}$. Although the conductivity of the graphene over a frequency range can be defined using (10) and (11), the patch conductivity of the operating frequency region for a patch element is influenced by its band structure due to Pauli's blocking effect [10], [25]. At low frequency range (within microwave and millimeter frequencies), the intraband transition process is dominant and the interband process can be ignored. However considering the graphene for its Fermi Dirac behavior with the minimum conductivity being achieved at its Fermi Dirac point as defined in (13), the maximum resistivity of the graphene sheet can be obtained using (14) [26] - [28]. Assuming graphene flake to have zero thickness, the conductivity can be derived using relations (10) and (11) with the unit of Siemens however graphene being one atom thick material with its thickness 'delta' of $0.335 \mathrm{~nm} \mathrm{[14]} \mathrm{its} \mathrm{volumetric}$ conductivity can be obtained using $\left(\sigma_{\text {inter }}+\sigma_{\text {intra }}\right) /$ delta. As the patch used in the antenna design of this paper has its thickness 'tp', the number of graphene layers ' $N$ ' can be obtained using tp/delta. The comparison of sheet resistance (an inverse of volumetric conductivity) along with the consideration of skin depth for copper with graphene shows only a small improvement in the copper conductivity. As per IPC 4562 specification, the resistivity of copper at room temperature is within the range of 1.68 $1.72 \mu \Omega \mathrm{cm}$. The pouring of copper patch to make a metallic part and considering its skin depth with $0.5 \mathrm{oz}$. thickness, the sheet resistance of the laid over copper can be obtained as $1.72 \mathrm{e}-8 / 17.5 \mathrm{e}-6=1 \mathrm{~m} \Omega / \mathrm{sq}$. The skin depth is defined as (12) [29]. The conductivity for the graphene 
is frequency dependent while the copper's conductivity is assumed constant over its frequency range with $\sigma=5.8 \mathrm{e} 7$. Considering the surface impedance plot as shown in Fig. 5 and the thickness of a graphene flake being $0.335 \mathrm{~nm}$, the mean surface impedance of the doped graphene flake (although it is a function of frequency) within $\mathrm{GHz}$ range at $0.1 \mathrm{mV}$ can be considered about $8.8 \Omega / \mathrm{sq}$ [2], [3] .

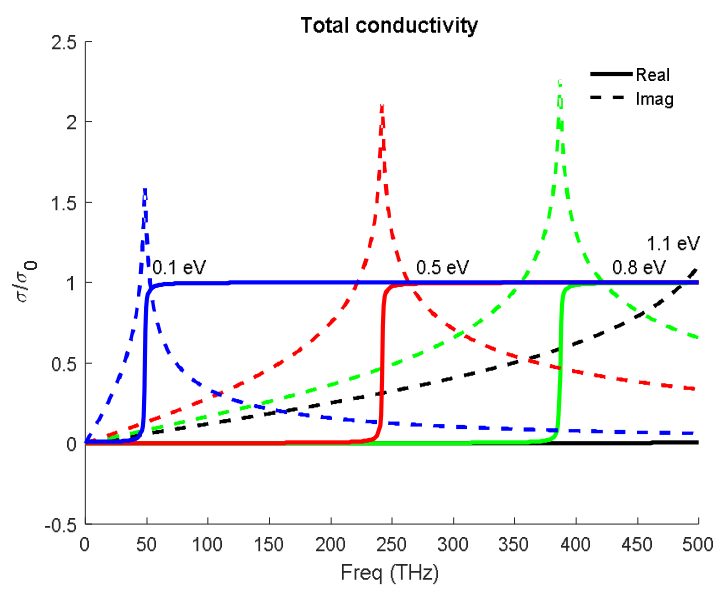

Fig. 4 Normalized total conductivity of graphene

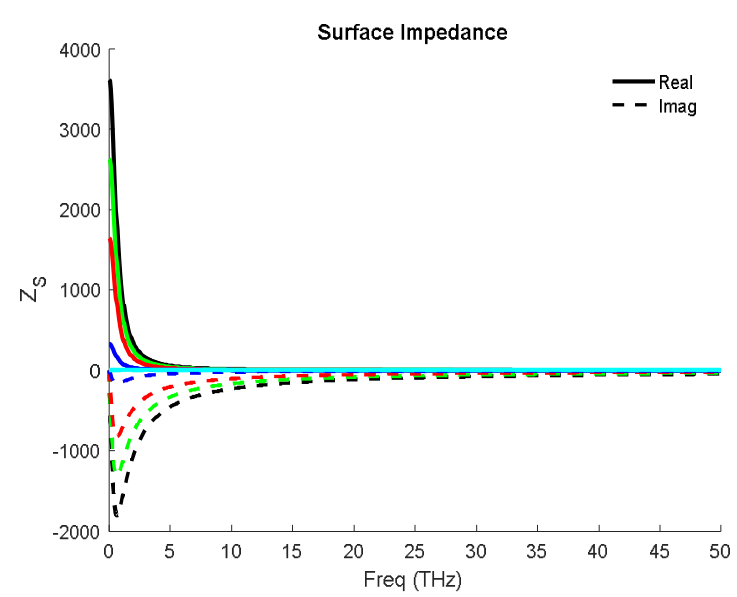

Fig. 5 Surface impedance of the graphene sheet

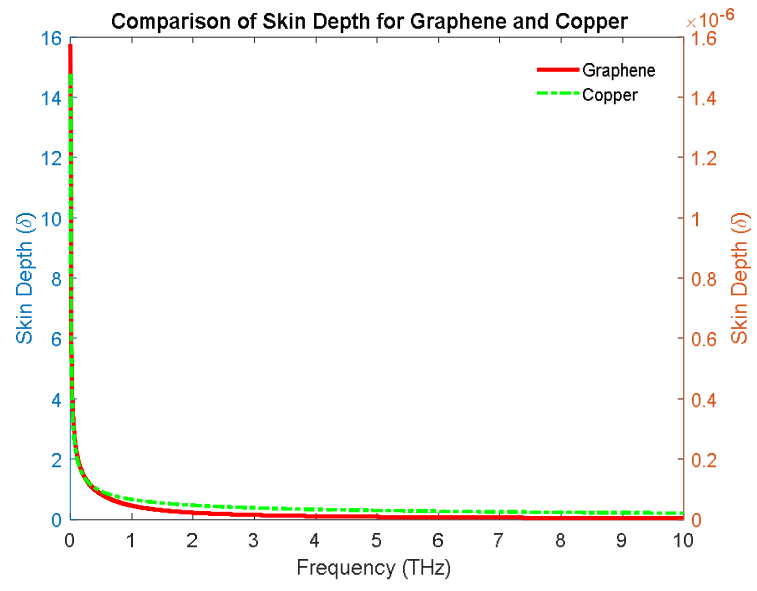

Fig. 6 Skin depth comparison for graphene and copper
With ' $\mathrm{N}$ ' layer thick graphene, its skin depth can be plotted using (12) and is shown in Fig. 6. Here the sheet resistance of graphene can be obtained as $8.8 / \mathrm{N} \Omega / \mathrm{sq}$. Thus with an increase in graphene layers deposit, this results in similar sheet resistance as of copper (within a factor of $10 \mathrm{~s}$ ) however presently there is manufacturing constraint for very thick layers of graphene flake. Using (10) with the operating frequency within micrometer and/or millimeter wave and $\tau$ in ps, the denominator term $(\omega-j 2 / \tau)$ can be approximated to a frequency independent term $(-\mathrm{j} 2 / \tau)$. The conductivity can be assumed to be independent of its operating frequency. The surface resistance $\left(R_{s}\right)$ of a single layer graphene sheet (with its thickness $0.335 \mathrm{~nm}$ [14]) can be obtained using $\left(R_{s}=\sigma_{s}^{-1}\right)$. With the development of multi-layer techniques for graphene and using a simplified resistance/conductance summation technique, the total conductivity of $\mathrm{N}$-layer surface is equal to $\mathrm{N}^{*} \sigma_{\mathrm{s}}$ or its total surface impedance is equal to $\left(\mathrm{N}^{*} \sigma_{\mathrm{s}}\right)^{-1}$ [10], [30], [31]. It is assumed that each and every graphene layer has the same relaxation time and Fermi energy.

$$
\begin{aligned}
& \sigma_{\text {dirac }}\left(\omega, \mu_{0}, \tau, T\right)=-j \frac{e^{2} k_{B} T 2 \ln 2}{\pi \hbar^{2}(\omega-j 2 / \tau)} \\
& R^{\text {dirac }}=\frac{2 \pi \hbar^{2} / \tau}{e^{2} k_{B} T 2 \ln 2}
\end{aligned}
$$

\section{II.2. Elliptical Patch Antenna}

An elliptical patch antenna as shown in Figs. 7 and 8 with its top and side view has been designed over a polyimide substrate (dielectric constant 3.5 and dielectric loss 0 ) with its thickness $1.6 \mathrm{~mm}$ to operate at $10.2 \mathrm{GHz}$. The elliptical patch is made of copper and doped graphene and has its $\mathrm{x}$ - direction radius ' $\mathrm{R}_{\mathrm{x}}$ ' of $25 \mathrm{~mm}$ while its $\mathrm{y}$ direction radius ' $\mathrm{R}_{\mathrm{y}}$ ' is $16 \mathrm{~mm}$.

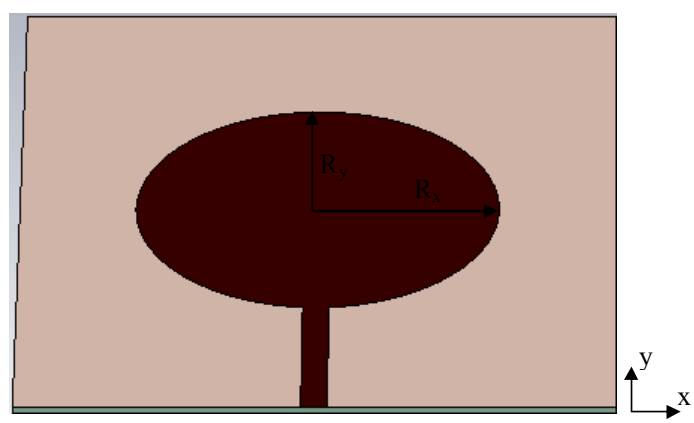

Fig. 7 Elliptical patch antenna (Top view)

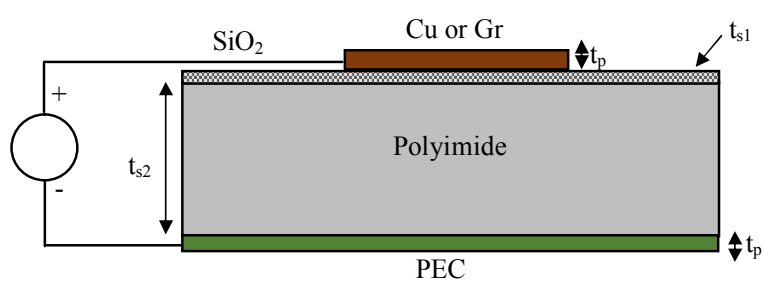

Fig. 8 Elliptical patch antenna (Side view) 
The patch is excited through a feed element and has a dimension of $3.6 \mathrm{~mm} \times 16 \mathrm{~mm}$. The thickness of the patch 'tp' is $6 \mu \mathrm{m}$ and is laid over $0.3 \mu \mathrm{m}$ thick $\mathrm{SiO}_{2}$ substrate (dielectric constant 11.5 and dielectric loss 0). The $\mathrm{SiO}_{2}$ substrate is in turn laid over a polyimide substrate with thickness 'ts' of $1.6 \mathrm{~mm}$. A ground plane of thickness 'tg' with $6 \mu \mathrm{m}$ has been designed with PEC for these two cases, copper patch and graphene patch antenna. The S11 parameter has been obtained for the copper and graphene patch and its comparison plot is shown in Fig. 9.

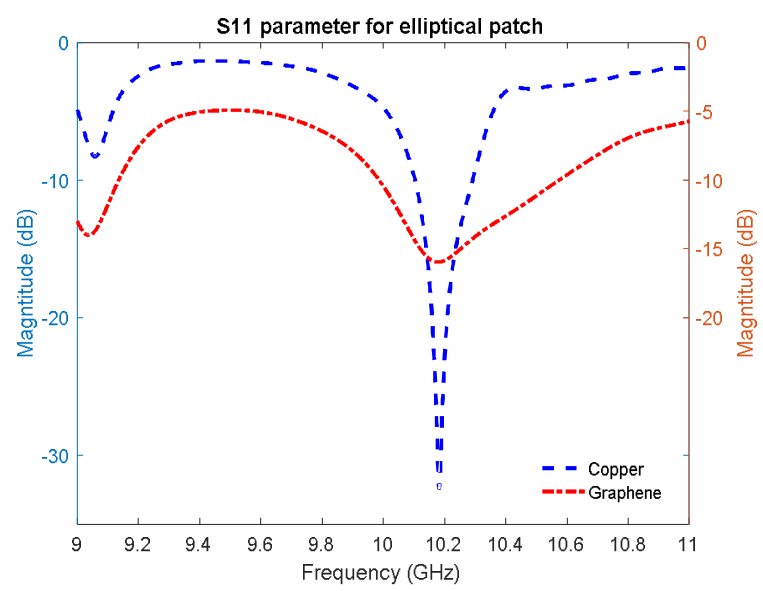

Fig. 9 S11 parameter comparison for Elliptical patch

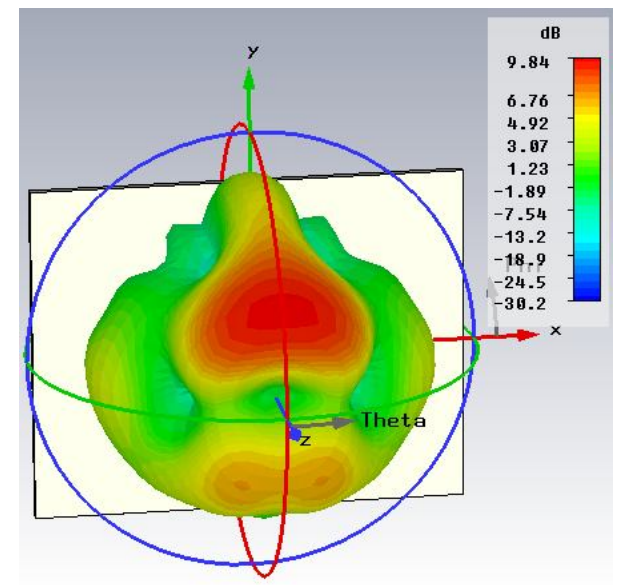

Fig. 10 Gain for Elliptical copper patch

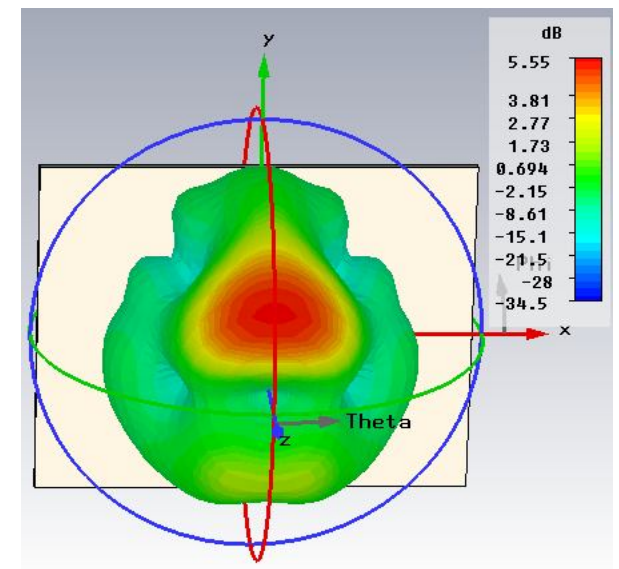

Fig. 11 Gain for Elliptical graphene patch
The gain for a copper and doped graphene patch antenna while fixing the DC bias voltage at $0.1 \mathrm{mV}$ have been obtained and are shown in Figs. 10 and 11.

\section{II.2.1 Spice circuit for Elliptical Patch}

S11 parameter plot can be obtained using 3D field solver tool however due to its resource consumption and its other difficulties such as specific knowledge and cost, it becomes important to obtain an electrical equivalence with spice circuit to get the similar result.

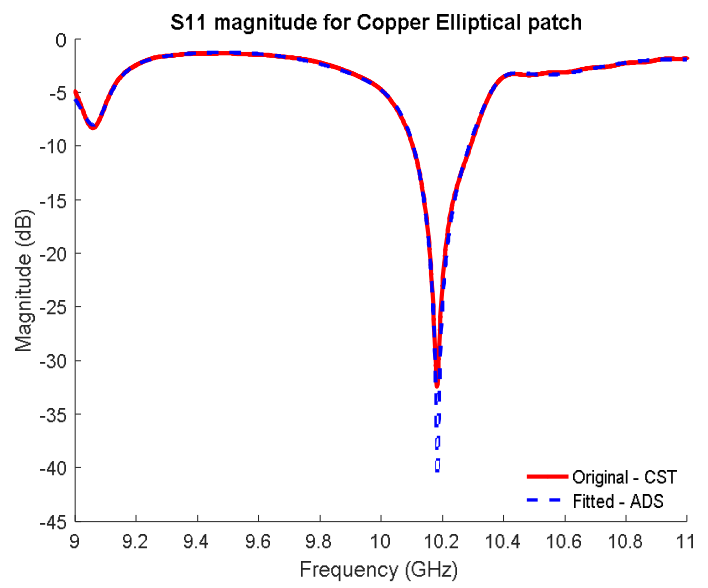

Fig. 12 S11 original and fitted magnitude for Copper Patch

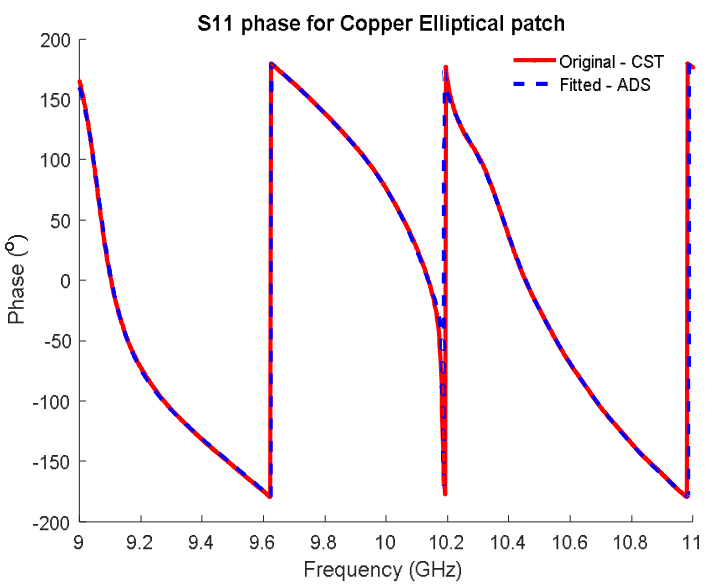

Fig. 13 S11 original and fitted phase for Copper Patch

TABLE I

RLC PARAMETERS FOR THE SPICE CIRCUIT

\begin{tabular}{lcll}
\hline $\begin{array}{l}\text { RLC } \\
\text { parameters }\end{array}$ & Stage 1 & \multicolumn{1}{c}{ Stage 2 } & Stage 3 \\
\hline $\mathrm{R}_{\mathrm{s}}(\mathrm{i})(\Omega)$ & 200.394372225428 & -370.144617750422 & 68.3171002749970 \\
$\mathrm{~L}(\mathrm{i})(\mathrm{nH})$ & & 24.2088313202814 & 7.17761406514047 \\
$\mathrm{C}(\mathrm{i})(\mathrm{fF})$ & & 12.0719174405403 & 28.7577980226560 \\
$\mathrm{Rp}(\mathrm{i})(\mathrm{k} \Omega)$ & & 5.22097960039867 & -3.98298271326505 \\
\hline
\end{tabular}

\begin{tabular}{llll}
\hline $\begin{array}{l}\text { RLC } \\
\text { parameters }\end{array}$ & Stage 4 & \multicolumn{1}{c}{ Stage 5 } & Stage 6 \\
\hline $\mathrm{R}_{\mathrm{s}}(\mathrm{i})(\Omega)$ & 29.3549414329264 & 7931.06222887171 & -140143.178627130 \\
$\mathrm{~L}(\mathrm{i})(\mathrm{nH})$ & 7.95138471347810 & 79.5077390754079 & -331.420407536658 \\
$\mathrm{C}(\mathrm{i})(\mathrm{fF})$ & 34.2999841784649 & 0.903980283334481 & -0.01653666104953 \\
$\mathrm{Rp}(\mathrm{i})(\mathrm{k} \Omega)$ & -9.24011913156274 & -11.2470788640509 & 143.450091566346 \\
\hline
\end{tabular}


The S- parameters of the copper and graphene patch has been converted into touchstone files for its import and conversion using ADS tool after satisfying the passivity condition. An equivalent RLC ladder circuit and fitted Sparameter magnitude and phase response for the copper patch are shown in Figs. 12 and 13. The RLC parametric values and its circuit has been obtained and are shown in Table I and Fig. 14.

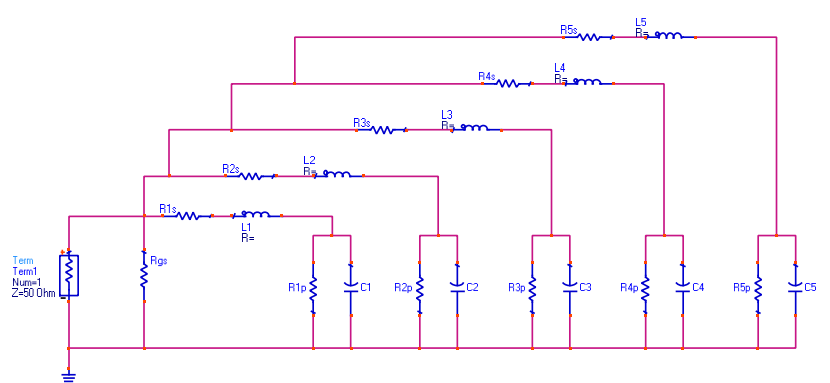

Fig. 14 Spice circuit for Copper Patch Antenna

Similarly an equivalent RLC ladder circuit and fitted Sparameters response for the graphene patch are shown in Figs. 15 and 16. while the obtained RLC parametric values and its circuit have been shown in Table II and Fig. 17.

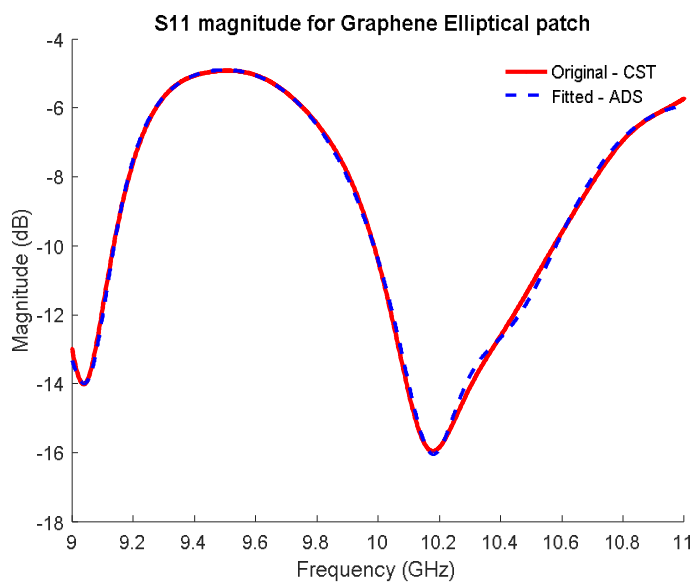

Fig. 15 S11 original and fitted magnitude for Graphene Patch

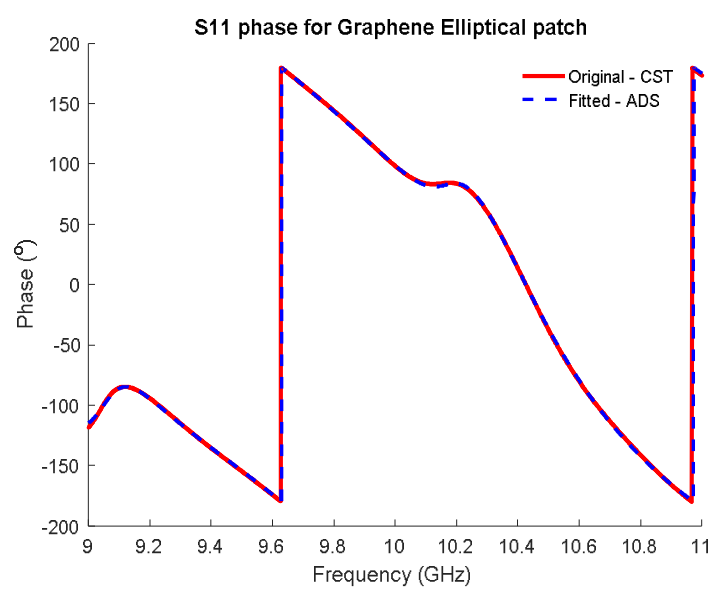

Fig. 16 S1 1 original and fitted phase for Graphene Patch
TABLE II

RLC PARAMETERS FOR THE SPICE CIRCUIT

\begin{tabular}{lcll}
\hline RLC & \multicolumn{1}{c}{ Stage 1 } & \multicolumn{1}{c}{ Stage 2 } & Stage 3 \\
parameters & & & \\
\hline $\mathrm{R}_{\mathrm{s}}(\mathrm{i})(\Omega)$ & 105.934037904607 & -4414.59574889389 & 198.389658336068 \\
$\mathrm{~L}(\mathrm{i})(\mathrm{nH})$ & & 51.2960730613653 & 8.14783402631080 \\
$\mathrm{C}(\mathrm{i})(\mathrm{fF})$ & & 1.79845400802354 & 23.2989187514182 \\
$\mathrm{Rp}(\mathrm{i})(\mathrm{k} \Omega)$ & & 6.28159698602455 & -1.95950171687396 \\
\hline
\end{tabular}

\begin{tabular}{lcc}
\hline $\begin{array}{l}\text { RLC } \\
\text { parameters }\end{array}$ & Stage 4 & Stage 5 \\
\hline $\mathrm{R}_{\mathrm{s}}(\mathrm{i})(\Omega)$ & 6.99215234028465 & -1375.34187589976 \\
$\mathrm{~L}(\mathrm{i})(\mathrm{nH})$ & 8.13870710466372 & 72.7258825756975 \\
$\mathrm{C}(\mathrm{i})(\mathrm{fF})$ & 33.6418391427814 & 3.03950691280753 \\
$\mathrm{Rp}(\mathrm{i})(\mathrm{k} \Omega)$ & 27.5463655221363 & 15.8304633044041 \\
\hline
\end{tabular}

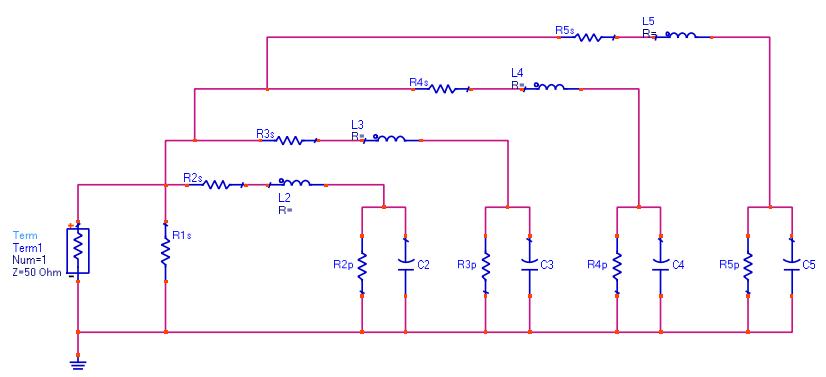

Fig. 17 Spice circuit for Graphene Patch Antenna

\section{II.3. Elliptical Patch Logo Antenna}

The Logo antenna with the elliptical patch shape as described in Section 3.1 has been used here for its dimension.

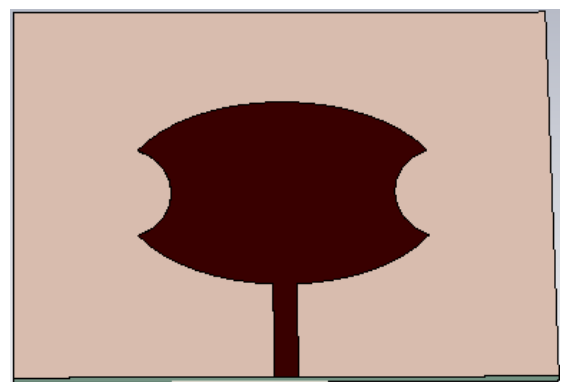

Fig. 18 Elliptical patch Logo antenna

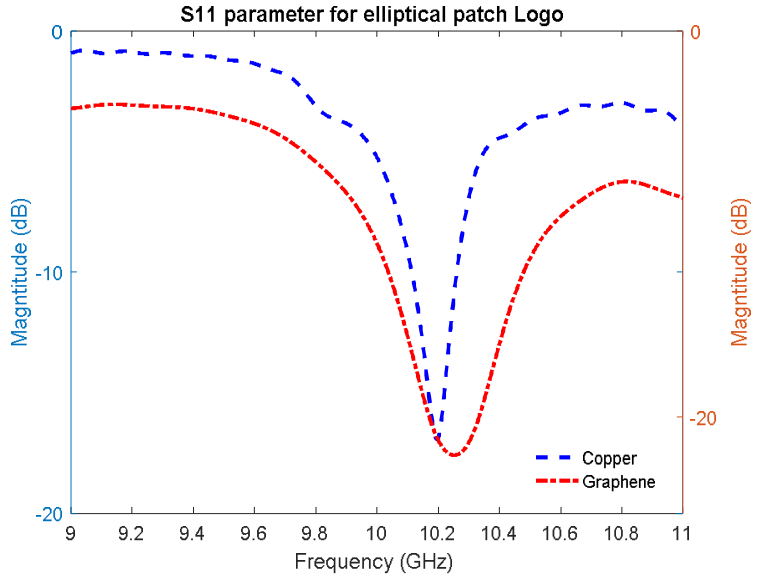

Fig. 19 S11 parameter comparison for Logo patch 
However to create a Logo, the left and right side periphery has been cut with $8 \mathrm{~mm}$ radius and is shown in Fig. 18. The comparison of S11 parameter for the copper and graphene patch Logo antenna has been obtained and is shown in Fig. 19. Similarly the gain for a copper and graphene patch antenna while fixing the DC bias voltage at $0.1 \mathrm{mV}$ have been obtained and are shown in Figs. 20 and 21.

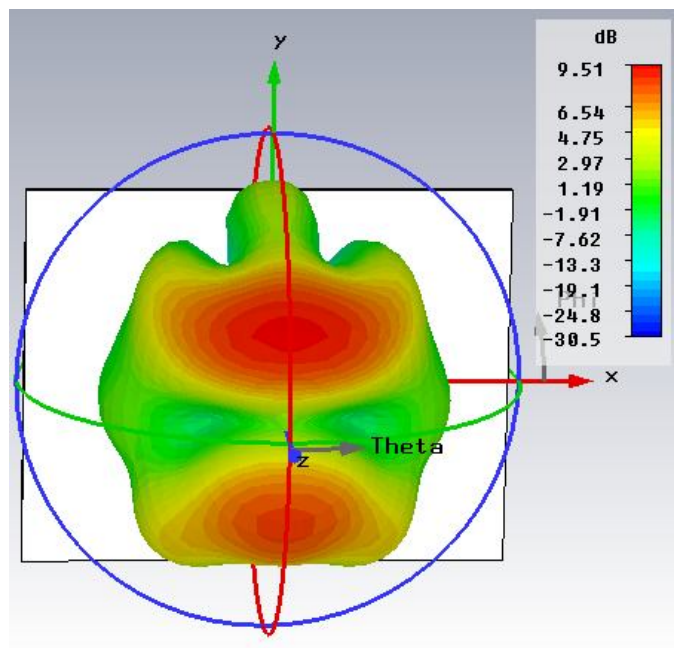

Fig. 20 Gain for Elliptical copper patch Logo

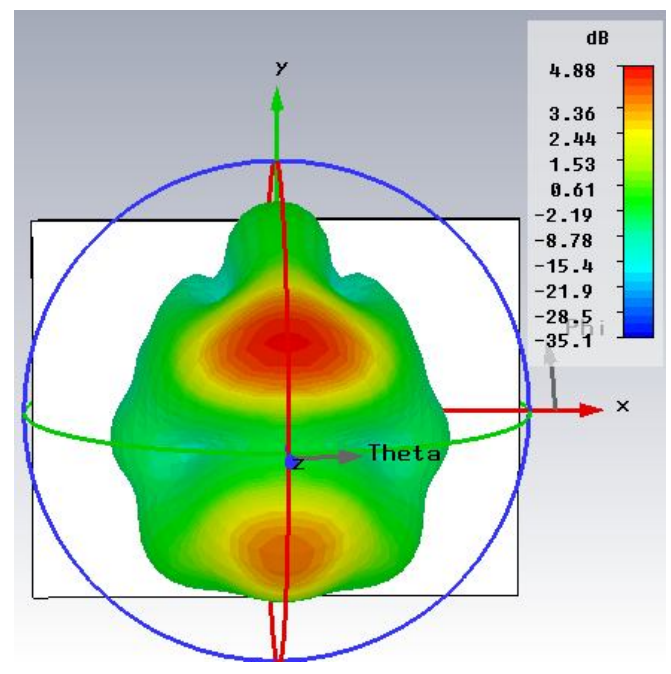

Fig. 21 Gain for Elliptical graphene patch Logo

\section{Results and Discussion}

Various antenna parameters such as S11 parameter, VSWR, Bandwidth, Gain, Angular width and efficiency have been obtained for the elliptical patch and elliptical patch Logo antenna with copper and graphene and are shown in Table III. As shown here, a metallic doping in the graphene patch element can provide similar results to a copper conductive media at $10 \mathrm{GHz}$ resonance with considerable bandwidth although the graphene patch shows a small shift in its resonant frequency and an increase in bandwidth. Similarly the gain and radiation efficiency for the graphene patch antenna is around $5 \mathrm{~dB}$ and $30 \%$ while the copper patch has a gain and radiation efficiency of about $10 \mathrm{~dB}$ and $95 \%$. Although there is some reduction in gain with a significant reduction in its radiation efficiency, still the graphene patch element provides reasonable radiation and total efficiency to work as antenna.

TABLE III

PATCH ANTENNA PARAMETERS

\begin{tabular}{|c|c|c|c|c|c|}
\hline & Patch & $\begin{array}{c}\text { Freq }\left(\mathbf{f}_{\mathbf{o}}\right) \\
\text { in } \mathbf{G H z}\end{array}$ & $\begin{array}{c}\text { Bandwidth } \\
\text { (MHz) }\end{array}$ & S11 (dB) & VSWR \\
\hline Elliptical & Copper & 10.184 & 188 & -32.26 & 1.05 \\
\hline patch & Graphene & 10.178 & 584 & -15.96 & 1.38 \\
\hline Elliptical & Copper & 10.191 & 146 & -16.87 & 1.33 \\
\hline \multirow[t]{2}{*}{ patch Logo } & Graphene & 10.252 & 605.7 & -21.99 & 1.17 \\
\hline & Patch & $\begin{array}{l}\text { Directivity } \\
\text { (dBi) }\end{array}$ & $\begin{array}{l}\text { Gain } \\
\text { (dB) }\end{array}$ & $\begin{array}{c}\text { Angular Ra } \\
\text { width Ef } \\
\left({ }^{\circ}\right)\end{array}$ & $\begin{array}{l}\text { adiation } \\
\text { fficiency } \\
(\%)\end{array}$ \\
\hline Elliptical & Copper & 10.00 & 9.84 & 33.0 & 95.86 \\
\hline patch & Graphene & 10.7 & 5.55 & 34.0 & 30.51 \\
\hline Elliptical & Copper & 9.71 & 9.51 & 28.7 & 95.56 \\
\hline patch Logo & Graphene & 9.96 & 4.88 & 32.5 & 31.07 \\
\hline
\end{tabular}

\section{Conclusion}

This paper provides an insight about the graphene based antenna design within $\mathrm{GHz}$ range. The efficiency and gain of any patch based antenna is highly dependent on its low surface impedance. Although graphene is more suitable within its $\mathrm{THz}$ frequency range due to its somewhat similar conductivity of other metals such as copper and the conductivity of the graphene becomes poor within $\mathrm{GHz}$ range, an increase in number of layers may provide an opportunity for an increase in conductivity and reduction in surface impedance. A simplified approach to increase the conductivity and decrease the surface impedance of graphene layers designed patch antenna has been used in this paper to show the application of graphene within $\mathrm{GHz}$ range. There has been a constraint in the manufacturing of multi-layer graphene with significant thickness so as to increase the conductivity considerably however the graphene flake can be mixed with other conducting metal particles to increase the conductivity and reduce the surface impedance within the $\mathrm{GHz}$ frequency range. A spice circuit in terms of RLC ladder network, which can provide an alternative circuit solution to $3 \mathrm{D}$ field solver tool, has been derived for its inclusion in any circuit solver tool. The derived circuit can be integrated with any IBIS/Spice circuit for providing the solution without relying on a 3D field solver tool.

\section{Acknowledgements}

This work was funded and completed as part of the UROP project supervision at CSIC, department of Engineering, University of Cambridge. 


\section{References}

[1] G. Deligeorgis, M. Dragoman, D. Neculoiu, D. Dragoman, G. Konstantinidis, A. Cismaru, and R. Plana, Microwave propagation in graphene, Appl. Phys. Lett., Volume 95, 2009, Pages 073107. doi: $10.1063 / 1.3202413$

[2] A. K. Geim, and K. S. Novoselov, The rise of graphene. Nature Mater. Volume 6, 2007, Pages 183-191. doi :10.1038/nmat1849

[3] I. Khrapach, F. Withers, T. H. Bointon, D. K. Polyushkin, W. L. Barnes, S. Russo and M. F. Craciun, Novel highly conductive and transparent graphene-based conductors, Adv. Mater., Volume 24, 2012, Pages 2844-2849. doi: 10.1002/adma.201200489

[4] T. H. Bointon, S. Russo and M. F. Craciun, Is graphene a good transparent electrode for photovoltaics and display applications?, IET Circuits Devices Syst., Volume 9, Issue 6, 2015, Pages 403-412. doi: 10.1049/iet-cds.2015.0121

[5] F. Schwierz, Graphene Transistors: Status, Prospects, and Problems, in Proceedings of the IEEE, Volume. 101, 2013, Pages 1567-1584. doi: 10.1109/JPROC.2013.2257633

[6] D. Akinwande; N. Petrone; and J. Hone, Two-Dimensional Flexible Nanoelectronics, Nat. Commun, Volume 5, 2014, Pages 5678. doi: $10.1038 / \mathrm{ncomms} 6678$

[7] G. Giovannetti, P. A. Khomyakov, G. Brocks, V. M. Karpan, J. van denBrink, and P. J. Kelly, Doping graphene with metal contacts, Phys. Rev. Lett., Volume 101, 2008, Pages 026803. doi: 10.1103/PhysRevLett.101.026803

[8] K. S. Novoselova, Graphene: The Magic of Flat Carbon, ECS Trans., Volume 19, 2009, Pages 3-7. doi: 10.1149/1.3119522

[9] A. H. C. Neto, N. M. R. Peres, K. S. Novoselov, and A. K. Geim, The electronic properties of graphene, Rev. Mod. Phys. Volume 81, 2009, Pages 109-162. doi: 10.1103/RevModPhys.81.109

[10] F. Bonaccorso, Z. Sun, T. Hasan and A. C. Ferrari, Graphene photonics and optoelectronics. Nature Photon., Volume 4, 2010, Pages 611-622. doi: 10.1038/nphoton.2010.186

[11] M. S. Dresselhaus, G. Dresselhaus, and P. C. Eklund, Science of fullerenes and carbon nanotubes. San Diego, London: Academic Press, 1996.

[12] P. R. Wallace, The Band Theory of Graphite, Physical Review, Volume 71, 1947, Pages 622. doi: 10.1103/PhysRev.71.622

[13] R. Saito, M. S. Dresselhaus, and G. Dresselhaus, Physical Properties of Carbon Nanotubes. London, UK: Imperial College Press, 2003.

[14] M. J. Allen, V.C. Tung and R. B. Kaner, Honeycomb carbon: a review of graphene, Chem Rev., Volume 110, 2009, Pages 132-45. doi: $10.1021 / \mathrm{cr} 900070 \mathrm{~d}$

[15] T. Stauber, N. M. Peres, and A. K. Geim, Optical conductivity of graphene in the visible region of the spectrum, Physical Review B, Volume 78, 2008, Pages 085432. doi: 10.1103/PhysRevB.78.085432

[16] S. A. Mikhailov and K. Ziegler. New electromagnetic mode in graphene. Physical Review Letters, Volume 99, 2007, Pages 016803. doi: 10.1103/PhysRevLett.99.016803

[17] G. W. Hanson, Dyadic Green's functions and guided surface waves for a surface conductivity model of graphene, J. Appl. Phys., Volume 103, 2008, Pages 064302. doi: 10.1063/1.2891452

[18] R. R. Nair, P. Blake, A. N. Grigorenko, K. S. Novoselov, T. J. Booth, T. Stauber, N. M. R. Peres, and A. K. Geim, Fine structure constant defines visual transparency of graphene. Science, Volume 320, 2008, Pages 1308. doi: 10.1126/science. 1156965

[19] X. Qin, J. Chen, C. Xie, N. Xu and J. Shi, A tunable THz dipole antenna based on graphene, 2016 IEEE MTT-S International Microwave Workshop Series on Advanced Materials and Processes for RF and THz Applications (IMWS-AMP), Chengdu, 2016, pp. 13. doi: 10.1109/IMWS-AMP.2016.7588365

[20] J. S. Gomez-Diaz and J. Perruisseau-Carrier, Microwave to THz properties of Graphene and potential antenna applications, 2012 International Symposium on Antennas and Propagation (ISAP), Nagoys, 2012, pp. 239-242.

[21] M. Qu, M. Rao, S. Li, and L. Deng, Tunable antenna radome based on graphene frequency selective surface, American Institute of Physics, Volume 7, 2017, Pages 095307. doi: 10.1063/1.5003020
[22] R. Wang, S. Raju, M. Chan, and L. J. Jiang, Low frequency behavior of CVD graphene from DC to $40 \mathrm{GHz}$, Progress In Electromagnetics Research C, Volume 71, 2017, Pages 1-7. doi:10.2528/PIERC16111901

[23] J. Perruisseau-Carrier, M. Tamagnone, J. S. Gomez-Diaz, and E. Carrasco, Graphene antennas: Can integration and reconfigurability compensate for the loss?, in European Microwave Conference (EuMC) (IEEE, 2013), pp. 369-372.

[24] M. Dragoman, D. Neculoiu1, A.-C. Bunea, G. Deligeorgis, M. Aldrigo, D. Vasilache, A. Dinescu, G. Konstantinidis, D. Mencarelli, L. Pierantoni, and M. Modreanu, A tunable microwave slot antenna based on graphene, Appl. Phys. Lett., Volume 106, 2015, Pages 153101. doi: http://dx.doi.org/10.1063/1.4917564

[25] L. M. Malard, K. F. Mak, A. H. C. Neto, N. M. R. Peres and T. F. Heinz, Observation of intra- and inter-band transitions in the transient optical response of graphene, New Journal of Physics, Volume 15, 2013, Pages 015009. doi: 10.1088/13672630/15/1/015009

[26] J. S. Gomez-Diaz, J. Perruisseau-Carrier, P. Sharma, and A. Ionescu, Non-contact characterization of graphene surface impedance at micro and millimeter waves, J. Appl. Phys., Volume 111, 2012, Pages 114908. https://doi.org/10.1063/1.4728183

[27] Y. W. Tan, Y. Zhang, K. Bolotin, Y. Zhao, S. Adam, E. H. Hwang, S. Das-Sarma, H. L. Stormer and P. Kim, Measurement of scattering rate and minimum conductivity in graphene, Phys. Rev. Lett., Volume 99, 2007, Pages 246803. doi: 10.1103/PhysRevLett.99.246803

[28] L. Hao, J. Gallop, S. Goniszewski1, A. Gregory, O. Shaforost, N. Klein and R. Yakimova, Non-contact method for measurement of the microwave conductivity of graphene, Appl. Phys. Lett. Volume 103, 2013, Pages 123103. doi: 10.1103/RevModPhys.81.109

[29] H. A. Wheeler, Formulas for the skin effect, Proc. IRE, Volume. 30, 1942, Pages 412-424. doi: 10.1109/JRPROC.1942.232015

[30] I. H. Baek, K. J. Ahn, B. J. Kang, S. Bae, B. H. Hong, D.-I. Yeom, K. Lee, Y. U. Jeong, and F. Rotermund, Terahertz transmission and sheet conductivity of randomly stacked multi-layer graphene, Appl. Phys. Lett., Volume 102, 2013, Pages 191109. doi: 10.1063/1.4805074

[31] B. Wu, H. M. Tuncer, M. Naeem, B. Yang, M. T. Cole, W. I. Milne, and Y. Hao, Experimental demonstration of a transparent graphene millimetre wave absorber with $28 \%$ fractional bandwidth at 140 $\mathrm{GHz}$ Scientific Reports, Volume 4, 2014, Pages 4130. doi: 10.1038/srep04130

[32] S. Sajal, B. D. Braaten, T. Tolstedt, S. Asif, and M. J. Schroeder, Design of a conformal Monopole antenna on a paper substrate using the properties of graphene-based conductors, Microwave and Optical Technology Letters, Volume 59, 2017, Pages 1279-1283. doi: $10.1002 / \mathrm{mop}$

[33] A. B. Kuzmenko, E. van Heumen, F. Carbone, and D. van der Marel. Universal optical conductance of graphite. Physical Review Letters, Volume 100, 2008, Pages 117401. doi: 10.1103/PhysRevLett.100.117401

\section{Authors' information}

${ }^{1}$ Department of Engineering, University of Cambridge, Trumpington Street, Cambridge, CB2 1PZ, UK

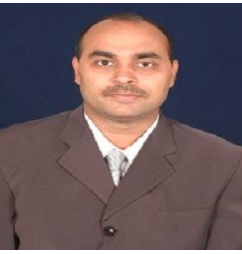

Varindra Kumar has obtained his Bachelor of Engineering in Electronics Engineering from National Institute of Technology (Formerly REC) Rourkela and Master of Technology in Electronics and Communication Engineering from Indian Institute of Technology, Varanasi (Formerly IT $\mathrm{BHU})$. Subsequently after working across various electronics design companies, he joined for $\mathrm{PhD}$ at the University of Nottingham in EMC Macromodeling. After finishing his $\mathrm{PhD}$ in the year 2014 he is working as Postdoc Research Associate at the department of Engineering, University of Cambridge. His research interest lies in electronics design, microprocessor architecture, sensor design, electromagnetic and antenna design etc. 